\title{
Applications of Certain Operators to the Classes of Analytic Functions Related to the Generalized Janowski Functions
}

\author{
Khalida Inayat Noor and Shujaat Ali Shah*
}

\begin{abstract}
We introduce certain subclasses of analytic functions related to the class of analytic, convex univalent functions. We discuss some results including inclusion relationships and invariance of the classes under convex convolution in terms of certain linear operators. Applications of these results associated with the generalized Janowski functions and conic domains are considered. Also, several radius problems are investigated.
\end{abstract}

\section{Introduction}

Let $\mathbf{A}$ be the class of analytic functions of the form

$$
f(z)=z+\sum_{n=2}^{\infty} a_{n} z^{n}
$$

in the open unit disk $E=\{z:|z|<1\}$. If $f$ and $g$ are analytic in $E$, we say that $f$ is subordinate to $g$, written $f \prec g$ or $f(z) \prec g(z)$, if there exists a Schwartz function $w$ in $E$ such that $f(z)=g(w(z))$.

The convolution or Hadamard product of two functions $f, g \in \mathbf{A}$ is denoted by $f * g$ and is defined as

$$
(f * g)(z)=z+\sum_{n=2}^{\infty} a_{n} b_{n} z^{n}, \quad z \in E
$$

Received: May 8, 2020; Accepted: May 17, 2020

2010 Mathematics Subject Classification: 30C45, 30C55.

Keywords and phrases: analytic functions, generalized Janowski functions, conic domains, Ruscheweyh differential operator, Noor integral operator, inclusion results, radius problems. 
Analytic functions $p$ in the class $P[A, B]$ can be defined by using subordination as follows [8].

Let $p$ be analytic in $E$ with $p(0)=1$. Then $p \in P[A, B]$, if and only if,

$$
p(z) \prec \frac{1+A z}{1+B z}, \quad-1 \leq B<A \leq 1, z \in E .
$$

Polatoglu et al. 23] introduced the class $P[A, B, \alpha]$ of analytic functions $p$, if and only if, there exists $p_{1} \in P[A, B]$ such that for $z \in E$,

$$
p(z)=(1-\alpha) p_{1}(z)+\alpha, \quad(0 \leq \alpha<1) .
$$

The class $P\left[A_{1}, B_{1}\right]$ of analytic functions $p$ with $p(z) \prec \frac{1+A_{1} z}{1+B_{1} z}, z \in E$, by using more general bilinear transformation $h(z)=\frac{1+A_{1} z}{1+B_{1} z}$, with $A_{1} \in \mathbb{C}$ (complex plane), $B_{1} \in[-1,0]$ and $A_{1} \neq B_{1}$.

If $A_{1}=[(1-\alpha) A+\alpha B]$ and $B_{1}=B$ for $0 \leq \alpha<1$, and $-1 \leq B<A \leq 1$, then the class $P\left[A_{1}, B_{1}\right]$ reduces to the class $P[A, B, \alpha]$, which is denoted by $P\left[A_{1}, B\right]$. See [13].

For $k \geqslant 0$, the conic domains $\Omega_{k}$, defined as;

$$
\Omega_{k}=\left\{u+i v: u>k \sqrt{(u-1)^{2}+v^{2}}\right\} .
$$

The domains $\Omega_{k}(k=0)$ represents right half plane, $\Omega_{k}(0<k<1)$ represents hyperbola, $\Omega_{k}(k=1)$ represents a parabola and $\Omega_{k}(k>1)$ represents an ellipse. The extremal functions for these conic regions are given as

$$
p_{k}(z)=\left\{\begin{array}{lr}
\frac{1+z}{1-z}, & k=0 \\
1+\frac{2}{\pi^{2}}\left(\log \frac{1+\sqrt{z}}{1-\sqrt{z}}\right)^{2}, & k=1 \\
1+\frac{2}{1-k^{2}}\left[\left(\frac{2}{\pi} \arccos k\right) \arctan h \sqrt{z}\right], & 0<k<1 \\
1+\frac{1}{k^{2}-1} \sin \left(\frac{\pi}{2 R(t)} \int_{0}^{\frac{u(z)}{\sqrt{t}}} \frac{1}{\sqrt{1-x^{2}} \sqrt{1-(t x)^{2}}} d x\right) & +\frac{1}{k^{2}-1}, k>1
\end{array}\right.
$$

where $u(z)=\frac{z-\sqrt{t}}{z-\sqrt{t z}}, t \in(0,1), z \in E$ and $z$ is chosen such that $k=$ $\cosh \left(\frac{\pi R^{\prime}(t)}{4 R(t)}\right), R(t)$ is Legendre's complete elliptic integral of the first kind and 
$R^{\prime}(t)$ is complementary integral of $R(t)$; see [9, 10] for more information. These conic regions are being studied by several authors. See [1, 15, 17, 21].

Let $f \in \mathbf{A}$ and $D^{m}: \mathbf{A} \rightarrow \mathbf{A}$ be the operator defined by

$$
D^{m} f(z)=\left\{\begin{array}{ll}
\frac{z}{(1-z)^{m+1}} * f(z) ; & m>-1 \\
\frac{z\left(z^{m-1} f(z)\right)^{m}}{m !} & m \in \mathbb{N}_{0}=\{0,1,2, \ldots\}
\end{array} .\right.
$$

Note that $D^{0} f(z)=f(z)$ and $D^{1} f(z)=z f^{\prime}(z)$. The operator $D^{m} f$ is called the Ruscheweyh derivative of $m$ th order of $f$ [24].

We can easily verify the following identity,

$$
z\left(D^{m} f\right)^{\prime}=(m+1) D^{m+1} f-m D^{m} f .
$$

Analogous to the Ruscheweyh derivative operator, Noor [16] and Noor and Noor [19] defined an operator as follows:

Let

$$
f_{m}(z)=\frac{z}{(1-z)^{m+1}}, \quad m \in \mathbb{N}_{0}=\{0,1,2, \ldots\}
$$

and let $f_{m}^{(-1)}$ be defined such that

$$
f_{m}(z) * f_{m}^{(-1)}(z)=\frac{z}{(1-z)} .
$$

Then, the operator $I_{m}: \mathbf{A} \rightarrow \mathbf{A}$ is defined by

$$
I_{m} f(z)=f_{m}^{(-1)}(z) * f(z)=\left(\frac{z}{(1-z)^{m+1}}\right)^{(-1)} * f(z) .
$$

The following identity can easily be verified,

$$
z\left(I_{m+1} f\right)^{\prime}=(m+1) I_{m} f-m I_{m+1} f .
$$

The multiplier transformation [6] $\mathcal{L}_{b}^{\delta}: \mathbf{A} \rightarrow \mathbf{A}$ is defined as follows:

$$
\mathcal{L}_{b}^{\delta} f(z)=\psi(\delta, b ; z) * f(z)=z+\sum_{n=2}^{\infty}\left(\frac{b+n}{b+1}\right)^{\delta} a_{n} z^{n}
$$

where $\psi(\delta, b ; z)=z+\sum_{n=2}^{\infty}\left(\frac{b+1}{b+n}\right)^{\delta} z^{n},(b>-1, \delta$ be real, $z \in E)$. 
One can easily verify the following identity,

$$
z\left(\mathcal{L}_{b}^{\delta} f(z)\right)^{\prime}=(1+b) \mathcal{L}_{b}^{\delta+1} f(z)-b \mathcal{L}_{b}^{\delta} f(z) .
$$

For further detail about above given operators, one may see [5, 6, 13, 16, 18, [19.

Dziok and Noor [7], introduced the concepts of some general classes as follows:

Let $\mathbf{A}_{\mathbf{0}}$ be the class of functions $f \in \mathbf{A}$ with $f(0)=1$. Assume that $\mu, \lambda$, $\ell$ be real parameters, $\mu \geq 0, \ell \geq 2$ and let $\Phi=(\phi, \varphi) \in \mathbf{A} \times \mathbf{A}, \xi \in \mathbf{A}$ and $H=\left(h_{1}, h_{2}\right)$, where $h_{i}(i=1,2)$ are analytic, univalent convex functions with $h_{i}(0)=1(i=1,2)$. Then

$$
\begin{gathered}
P(h)=\left\{q \in \mathbf{A}_{\mathbf{0}}: q \prec h\right\}, \\
P_{\mu}(H)=\left\{\mu q_{1}+(1-\mu) q_{2}: q_{1} \prec h_{1}, q_{2} \prec h_{2}\right\}, \\
P_{\mu}((h, h))=P_{\mu}(h) \text { and } P_{\mu}\left(\frac{1+z}{1-z}\right)=P_{\ell}, \quad\left(\mu=\frac{\ell}{4}+\frac{1}{2}\right)
\end{gathered}
$$

where $P_{\ell}$ is the class introduced and studied by Pinchuk [22].

A function $f \in \mathbf{A}$ is said to be in the class $M_{\mu}^{\lambda}(\Phi, \xi, H)$, if and only if, $J_{\lambda}(f(z)) \in P_{\mu}(H)$, where

$$
J_{\lambda}(f(z))=(1-\lambda) \frac{\phi * \xi * f}{\varphi * \xi * f}+\lambda \frac{\phi * f}{\varphi * f} .
$$

We denote by $W_{\mu}(\Phi, \xi, H)=M_{\mu}^{0}(\Phi, \xi, H)$, the class of functions $f \in \mathbf{A}$ such that

$$
\frac{\phi * \xi * f}{\varphi * \xi * f} \in P_{\mu}(H)
$$

Moreover, let us define

$$
S^{*}(\varphi, \xi, h)=W_{1}\left(\left(z \varphi^{\prime}, \varphi\right), \xi, h\right) \text { and } C(\varphi, \xi, h)=W_{1}\left(\left(\varphi_{2}, \varphi_{1}\right), \xi, h\right),
$$

where $\varphi_{1}(z)=z \varphi^{\prime}(z)$ and $\varphi_{2}(z)=z \varphi_{1}^{\prime}$.

Definition 1. A function $f \in \mathbf{A}$ is in the class $S^{*}(\varphi, \xi, h)$, if and only if,

$$
\frac{z(\varphi * \xi * f)^{\prime}}{(\varphi * \xi * f)} \in P(h)
$$


Definition 2. A function $f \in \mathbf{A}$ is in the class $C(\varphi, \xi, h)$, if and only if,

$$
\frac{\left(z(\varphi * \xi * f)^{\prime}\right)^{\prime}}{(\varphi * \xi * f)^{\prime}} \in P(h) \text {. }
$$

For different choices of $\varphi, \xi$ and $h$, we will obtain well-known classes, see [4, 13, 14, 20, 27].

It is noted that

$$
f \in C(\varphi, \xi, h) \Leftrightarrow z f^{\prime} \in S^{*}(\varphi, \xi, h)
$$

\section{Preliminary Results}

Lemma 1 ([12]). Let $h$ be analytic, univalent convex function in $E$ with $h(0)=1$ and $\operatorname{Re}(\gamma h(z)+\sigma)>0, \sigma, \gamma \in \mathbb{C}$ and $\gamma \neq 0$. If $p(z)$ is analytic in $E$ and $p(0)=h(0)$, then

$$
\left\{p(z)+\frac{z p^{\prime}(z)}{\gamma p(z)+\sigma}\right\} \prec h(z),
$$

implies $p(z) \prec q(z) \prec h(z)$, where $q(z)$ is best dominant and is given as,

$$
q(z)=\left[\left\{\int_{0}^{1}\left(\exp \int_{t}^{t z} \frac{h(u)-1}{u} d u\right) d t\right\}^{-1}-\frac{\sigma}{\gamma}\right] .
$$

Lemma $2([26])$. Let $p$ be an analytic function in $E$ with $p(0)=1$ and $\operatorname{Re}\{p(z)\}>$ $0, z \in E$. Then, for $\vartheta>0$ and $\nu \neq-1$ (complex),

$$
\begin{gathered}
\operatorname{Re}\left\{p(z)+\frac{\vartheta z p^{\prime}(z)}{p(z)+\nu}\right\}>0, \text { for }|z|<r_{0}, \text { where } r_{0} \text { is given by } \\
r_{0}=\frac{|\nu+1|}{\sqrt{s+\sqrt{s^{2}-\left|\nu^{2}-1\right|^{2}}}}, \quad s=2(\vartheta+1)^{2}+|\nu|^{2}-1
\end{gathered}
$$

and this radius is best possible.

Lemma 3 ([25]). If $f \in C, g \in S^{*}$, then for each $h$ analytic in $E$ with $h(0)=1$,

$$
\frac{(f * h g)(E)}{(f * g)(E)} \subset \overline{C o} h(E),
$$

where $\overline{C o} h(E)$ denotes the convex hull of $h(E)$. 


\section{Main Results}

We assume throughout this paper $k \geq 0, b>-1, \delta$ be a real, $m \in \mathbb{N}_{0}=$ $\{0,1,2,3, \ldots\}, A_{1}=[(1-\alpha) A+\alpha B], B_{1}=B,(-1 \leq B<A \leq 1)$ and $z \in E$, unless otherwise stated.

Theorem 1. Let $\operatorname{Re}\{h(z)+b\}>0$ and $\operatorname{Re}\{h(z)+m\}>0$. Then

$$
\begin{aligned}
S^{*}\left(\psi(\delta+1, b ; z), f_{m}^{(-1)}, h\right) & \subset S^{*}\left(\psi(\delta, b ; z), f_{m}^{(-1)}, h\right) \\
& \subset S^{*}\left(\psi(\delta, b ; z), f_{m+1}^{(-1)}, h\right) .
\end{aligned}
$$

Proof. Let $f \in S^{*}\left(\psi(\delta+1, b ; z), f_{m}^{(-1)}, h\right)$.

For $I_{m} f(z)=f_{m}^{(-1)}(z) * f(z)$, we set

$$
\frac{z\left[\mathcal{L}_{a}^{\delta}\left(I_{m} f(z)\right)\right]^{\prime}}{\left[\mathcal{L}_{a}^{\delta}\left(I_{m} f(z)\right)\right]}=p(z),
$$

where $p(z)$ is analytic with $p(0)=1$.

Using identity (1.7) and (3.1), we have

$$
(1+b) \frac{\mathcal{L}_{b}^{\delta+1}\left(I_{m} f(z)\right)}{\mathcal{L}_{b}^{\delta}\left(I_{m} f(z)\right)}=p(z)+b .
$$

Logarithmic differentiating both sides, to get

$$
\frac{z\left[\mathcal{L}_{b}^{\delta+1}\left(I_{m} f(z)\right)\right]^{\prime}}{\mathcal{L}_{b}^{\delta+1}\left(I_{m} f(z)\right)}=p(z)+\frac{z p^{\prime}(z)}{p(z)+b} .
$$

Since $f \in S^{*}\left(\psi(\delta+1, b ; z), f_{m}^{(-1)}, h\right)$, so from (3.2) we have

$$
p(z)+\frac{z p^{\prime}(z)}{p(z)+b} \prec h(z) .
$$

By applying Lemma 1, it conclude that, $p(z) \prec h(z)$ and consequently,

$$
\frac{z\left[\mathcal{L}_{a}^{\delta}\left(I_{m} f(z)\right)\right]^{\prime}}{\left[\mathcal{L}_{a}^{\delta}\left(I_{m} f(z)\right)\right]} \prec h(z) .
$$

This implies $f(z) \in S^{*}\left(\psi(\delta, b ; z), f_{m}^{(-1)}, h\right)$. 
Now suppose that $f \in S^{*}\left(\psi(\delta, b ; z), f_{m}^{(-1)}, h\right)$.

For $\mathcal{L}_{b}^{\delta} f(z)=\psi(\delta, b ; z) * f(z)$, we set

$$
\frac{z\left[I_{m+1}\left(\mathcal{L}_{a}^{\delta} f(z)\right)\right]^{\prime}}{I_{m+1}\left(\mathcal{L}_{a}^{\delta} f(z)\right)}=Q(z),
$$

where $Q(z)$ is analytic with $Q(0)=1$.

From (1.6) and (3.4), we have

$$
(1+m) \frac{I_{m}\left(\mathcal{L}_{b}^{\delta} f(z)\right)}{I_{m+1}\left(\mathcal{L}_{b}^{\delta} f(z)\right)}=Q(z)+m .
$$

Logarithmic differentiating both sides, to get

$$
\frac{z\left[I_{m}\left(\mathcal{L}_{a}^{\delta} f(z)\right)\right]^{\prime}}{I_{m}\left(\mathcal{L}_{a}^{\delta} f(z)\right)}=Q(z)+\frac{z Q^{\prime}(z)}{Q(z)+m} .
$$

Since $f \in S^{*}\left(\psi(\delta, b ; z), f_{m}^{(-1)}, h\right)$, so from (3.5) we have

$$
Q(z)+\frac{z Q^{\prime}(z)}{Q(z)+m} \prec h(z) .
$$

By applying Lemma 1, it conclude that, $Q(z) \prec h(z)$ and consequently,

$$
\frac{z\left[I_{m+1}\left(\mathcal{L}_{a}^{\delta} f(z)\right)\right]^{\prime}}{I_{m+1}\left(\mathcal{L}_{a}^{\delta} f(z)\right)} \prec h(z) .
$$

This implies $f(z) \in S^{*}\left(\psi(\delta, b ; z), f_{m+1}^{(-1)}, h\right)$.

Theorem 2. Let $\operatorname{Re}\{h(z)+b\}>0$ and $\operatorname{Re}\{h(z)+m\}>0$. Then

$$
\begin{aligned}
C\left(\psi(\delta+1, b ; z), f_{m}^{(-1)}, h\right) & \subset C\left(\psi(\delta, b ; z), f_{m}^{(-1)}, h\right) \\
& \subset C\left(\psi(\delta, b ; z), f_{m+1}^{(-1)}, h\right) .
\end{aligned}
$$

Proof. Let

$$
\begin{aligned}
f & \in C\left(\psi(\delta+1, b ; z), f_{m}^{(-1)}, h\right) . \\
& \Leftrightarrow z f^{\prime} \in S^{*}\left(\psi(\delta+1, b ; z), f_{m}^{(-1)}, h\right), \\
& \Rightarrow z f^{\prime} \in S^{*}\left(\psi(\delta, b ; z), f_{m}^{(-1)}, h\right), \\
& \Leftrightarrow f \in C\left(\psi(\delta, b ; z), f_{m}^{(-1)}, h\right) .
\end{aligned}
$$

Similarly, we can prove $C\left(\psi(\delta, b ; z), f_{m}^{(-1)}, h\right) \subset C\left(\psi(\delta, b ; z), f_{m+1}^{(-1)}, h\right)$. 
We can deduce some main results as corollaries of the above theorems for different values of $h(z)$ given as bellow;

(a) $h(z)=\frac{1+A_{1} z}{1+B z}$.

(b) $h(z)=p_{k}(z)$, where $p_{k}(z)$ is defined by (1.4).

Theorem 3. Let $f \in S^{*}(\varphi, \xi, h)$ and $g$ be any convex univalent functions in $E$. Then $f * g \in S^{*}(\varphi, \xi, h)$.

Proof. Let $f \in S^{*}(\varphi, \xi, h)$. Then for $F=\xi * f$.

Consider

$$
\begin{aligned}
\frac{z(\varphi * \xi *(g * f))^{\prime}}{(\varphi * \xi *(g * f))} & =\frac{z(g *(\varphi * F))^{\prime}}{(g *(\varphi * F))} \\
& =\frac{g * z(\varphi * F)^{\prime}}{(g *(\varphi * F))} \\
& =\frac{g * \frac{z(\varphi * F)^{\prime}}{(\varphi * F)}(\varphi * F)}{(g *(\varphi * F))} .
\end{aligned}
$$

Since $\varphi * F \in S^{*}(h) \subset S^{*}$, so by lemma 3 , it follows that $f * g \in S^{*}(\varphi, \xi, h)$.

Theorem 4. Let $f \in C(\varphi, \xi, h)$ and $g$ be any convex univalent functions in $E$. Then $f * g \in C(\varphi, \xi, h)$.

Proof. We can easily prove this result by using Theorem 3 along with relation (1.8).

We can deduce some special cases for the Theorem 3 and Theorem 4, for different choices of $\varphi, \xi$ and $h$ as given below:

(i) If $\varphi(z)=\psi(\delta, b ; z), \xi(z)=f_{m}^{(-1)}(z)$ and $h$ is analytic, univalent convex function.

(ii) If $\varphi(z)=f_{m}(z), \xi(z)=\frac{z}{(1-z)}$ and $h(z)$ is analytic, univalent convex function.

(iii) If $\varphi, \xi \in \mathbf{A}$ and $h(z)=\frac{1+A_{1} z}{1+B z}$.

(iv) $\varphi, \xi \in \mathbf{A}$ and $h(z)=p_{k}(z)$, where $p_{k}(z)$ is defined by (1.4).

We can apply Theorem 3 and Theorem 4 to prove Integral preserving properties for the classes $S^{*}(\varphi, \xi, h)$ and $C(\varphi, \xi, h)$. 
Corollary 1. The class $S^{*}(\varphi, \xi, h)$ and $C(\varphi, \xi, h)$ is closed under the following operators.

(i) $f_{1}(z)=\int_{0}^{z} \frac{f(t)}{t} d t$.

(ii) $f_{2}(z)=\frac{2}{z} \int_{0}^{z} f(t) d t, \quad$ (Libera's operator [11]).

(iii) $f_{3}(z)=\int_{0}^{z} \frac{f(t)-f(x t)}{t-x t} d t, \quad|x| \leq 1, x \neq 1$.

(iv) $f_{4}(z)=\frac{c+1}{z^{c}} \int_{0}^{z} t^{c-1} f(t), \quad R e(c) \geq 0$, (Generalized Bernardi operator [3]).

Proof. We may write, $f_{i}(z)=f(z) * \phi_{i}(z)$, where $\phi_{i}(z), i=1,2,3,4$, are convex and given by

$$
\begin{aligned}
& \phi_{1}(z)=-\log (1-z)=\sum_{n=1}^{\infty} \frac{1}{n} z^{n} \\
& \phi_{2}(z)=\frac{-2[z-\log (1-z)]}{z}=\sum_{n=1}^{\infty} \frac{2}{n+1} z^{n}, \\
& \phi_{3}(z)=\frac{1}{1-x} \log \left(\frac{1-x z}{1-z}\right)=\sum_{n=1}^{\infty} \frac{1-x^{n}}{(1-x)^{n}} z^{n}, \quad|x| \leq 1, x \neq 1, \\
& \phi_{4}(z)=\sum_{n=1}^{\infty} \frac{1+c}{n+c} z^{n}, \quad \operatorname{Re}(c) \geq 0 .
\end{aligned}
$$

The proof follows easily by using Theorem 3 and Theorem 4 .

Now, we discuss some radius problems as follows:

Theorem 5. Let $f \in S^{*}\left(\psi(\delta, b ; z), f_{m}^{(-1)}, \frac{1+A_{1} z}{1+B z}\right)$. Then

$$
\begin{gathered}
f \in S^{*}\left(\psi(\delta+1, b ; z), f_{m}^{(-1)}, \frac{1+A_{1} z}{1+B z}\right), \text { for }|z|<r_{\delta}, \text { where } \\
r_{\delta}=\frac{2(1+\delta)}{L+\sqrt{L^{2}-4 M}},
\end{gathered}
$$

where $L=3 A_{1}^{2}+\delta\left(A_{1}+B\right)-B, \quad M=(1+\delta)\left(A_{1}^{2}+\delta A_{1} B\right)$. The value of $r_{\delta}$ is sharp.

Proof. Let $f \in S^{*}\left(\psi(\delta, b ; z), f_{m}^{(-1)}, \frac{1+A_{1} z}{1+B z}\right)$. Then, for $I_{m} f(z)=f_{m}^{(-1)}(z) * f(z)$

$$
\frac{z\left[\mathcal{L}_{a}^{\delta}\left(I_{m} f(z)\right)\right]^{\prime}}{\left[\mathcal{L}_{a}^{\delta}\left(I_{m} f(z)\right)\right]}=p(z),
$$


where $p(z)$ is analytic with $p(0)=1$. Using identity (1.7) and (3.8), we have

$$
(1+b) \frac{\mathcal{L}_{b}^{\delta+1}\left(I_{m} f(z)\right)}{\mathcal{L}_{b}^{\delta}\left(I_{m} f(z)\right)}=p(z)+b .
$$

Logarithmic differentiating both sides, to get

$$
\frac{z\left[\mathcal{L}_{b}^{\delta+1}\left(I_{m} f(z)\right)\right]^{\prime}}{\mathcal{L}_{b}^{\delta+1}\left(I_{m} f(z)\right)}=p(z)+\frac{z p^{\prime}(z)}{p(z)+b} .
$$

Since $\frac{1-A_{1} r}{1-B r} \leq \operatorname{Rep}(z) \leq \frac{1+A_{1} r}{1+B r}$ and $\left|\frac{z p^{\prime}(z)}{p(z)}\right| \geq \frac{\left(A_{1}-B\right) r}{\left(1-A_{1} r\right)(1-B r)}$, (see [2, 23]), then (3.9) implies,

$$
\operatorname{Re}\left(p(z)+\frac{z p^{\prime}(z)}{p(z)+b}\right) \geq \operatorname{Rep}(z)\left[\frac{\left(1-A_{1} r\right)\left\{\left(1-A_{1} r\right)+\delta(1-B r)\right\}-\left(A_{1}-B\right) r}{\left(1-A_{1} r\right)\left\{\left(1-A_{1} r\right)+\delta(1-B r)\right\}}\right] .
$$

The right hand side of above inequality is positive, for $|z|<r_{\delta}$, where $r_{\delta}$ is given by (3.7).

Corollary 2. Let $f \in S^{*}\left(\psi(\delta, b ; z), f_{m}^{(-1)}, \frac{1+A z}{1+B z}\right)$. Then $f \in S^{*}\left(\psi(\delta+1, b ; z), f_{m}^{(-1)}, \frac{1+A z}{1+B z}\right)$, for $|z|<r_{\delta}$, where

$$
r_{\delta}=\frac{2(1+\delta)}{L+\sqrt{L^{2}-4 M}},
$$

where $L=3 A^{2}+\delta(A+B)-B, \quad M=(1+\delta)\left(A^{2}+\delta A B\right)$. The value of $r_{\delta}$ is sharp.

Corollary 3. Let $f \in S^{*}\left(\psi(\delta, b ; z), f_{m}^{(-1)}, \frac{1+z}{1-z}\right)$. Then $f \in S^{*}\left(\psi(\delta+1, b ; z), f_{m}^{(-1)}, \frac{1+z}{1-z}\right)$, for $|z|<r_{\delta}$, where

$$
r_{\delta}=\frac{(1+\delta)}{2+\sqrt{3+\delta^{2}}} .
$$

The value of $r_{\delta}$ is sharp. 
Theorem 6. Let $F_{c} \in S^{*}\left(\psi(\delta, b ; z), f_{m}, \frac{1+A_{1} z}{1+B z}\right)$, where $F_{c}$ is defined by

$$
F_{c}=\frac{c+1}{z^{c}} \int_{0}^{z} t^{c-1} f(t) .
$$

Then

$\mathcal{L}_{a}^{\delta}\left(D^{m} f(z)\right) \in S^{*}\left(\frac{1+(1-2 \beta) z}{1-z}\right)$, for $|z|<r_{\beta}$, where

$$
r_{\beta}=\frac{|\nu+1|}{\sqrt{s+\sqrt{s^{2}-\left|\nu^{2}-1\right|^{2}}}},
$$

where $s=2(\vartheta+1)^{2}+|\nu|^{2}-1, \quad \vartheta=\frac{1}{1-\beta}, \quad \nu=\frac{\beta+c}{1-\beta}, \quad \beta=\frac{1-A_{1}}{1-B}$. The value of $r_{\beta}$ is sharp.

Proof. Let $F_{c} \in S^{*}\left(\psi(\delta, b ; z), f_{m}, \frac{1+A_{1} z}{1+B z}\right)$. Then for $D^{m} F_{c}(z)=f_{m}(z) * F_{c}(z)$,

$$
p(z)=\frac{z\left[\mathcal{L}_{a}^{\delta}\left(D^{m} F_{c}(z)\right)\right]^{\prime}}{\left[\mathcal{L}_{a}^{\delta}\left(D^{m} F_{c}(z)\right)\right]} \in P\left(\frac{1+A_{1} z}{1+B z}\right),
$$

where $p(z)$ is analytic with $p(0)=1$.

Since $F_{c}=\frac{c+1}{z^{c}} \int_{0}^{z} t^{c-1} f(t)$, so by differentiating, we have

$$
\begin{gathered}
\left(z^{c} F_{c}(z)\right)^{\prime}=(1+c) z^{c-1} f(z), \\
z F_{c}^{\prime}(z)+c F_{c}(z)=(1+c) f(z) .
\end{gathered}
$$

Taking convolution both sides of the above equation by $\psi(\delta, b ; z) * f_{m}$, to get

$$
\begin{gathered}
z\left[\mathcal{L}_{a}^{\delta}\left(D^{m} F_{c}(z)\right)\right]^{\prime}+c\left[\mathcal{L}_{a}^{\delta}\left(D^{m} F_{c}(z)\right)\right]=(1+c) \mathcal{L}_{a}^{\delta}\left(D^{m} f(z)\right), \\
\frac{z\left[\mathcal{L}_{a}^{\delta}\left(D^{m} F_{c}(z)\right)\right]^{\prime}}{\mathcal{L}_{a}^{\delta}\left(D^{m} F_{c}(z)\right)}+c=(1+c) \frac{\mathcal{L}_{a}^{\delta}\left(D^{m} f(z)\right)}{\mathcal{L}_{a}^{\delta}\left(D^{m} F_{c}(z)\right)}, \\
(1+c) \frac{\mathcal{L}_{a}^{\delta}\left(D^{m} f(z)\right)}{\mathcal{L}_{a}^{\delta}\left(D^{m} F_{c}(z)\right)}=p(z)+c . \quad \quad(\text { by }(3.11))
\end{gathered}
$$

Logarithmic differentiating both sides, we get

$$
\frac{z\left[\mathcal{L}_{a}^{\delta}\left(I_{m} f(z)\right)\right]^{\prime}}{\mathcal{L}_{a}^{\delta}\left(I_{m} f(z)\right)}=p(z)+\frac{z p^{\prime}(z)}{p(z)+c} .
$$


Since $p(z) \in P\left(\frac{1+A_{1} z}{1+B z}\right) \subset P\left(\frac{1+(1-2 \beta) z}{1-z}\right)$, so

$$
p(z)=(1-\beta) p_{1}(z)+\beta, \quad p_{1} \in P,
$$

where $\beta=\frac{1-A_{1}}{1-B}$.

From (3.12) and (3.13), we have

$$
\frac{1}{(1-\beta)}\left[\frac{z\left[\mathcal{L}_{a}^{\delta}\left(D^{m} f(z)\right)\right]^{\prime}}{\mathcal{L}_{a}^{\delta}\left(D^{m} f(z)\right)}-\beta\right]=p_{1}(z)+\frac{\frac{1}{(1-\beta)} z p_{1}^{\prime}(z)}{p_{1}(z)+\frac{(c+\beta)}{(1-\beta)}} .
$$

Take $\vartheta=\frac{1}{1-\beta}>0$ and $\nu=\frac{\beta+c}{1-\beta} \neq-1$, then by applying Lemma 2, we conclude that

$$
\frac{1}{(1-\beta)}\left[\frac{z\left[\mathcal{L}_{a}^{\delta}\left(D^{m} f(z)\right)\right]^{\prime}}{\mathcal{L}_{a}^{\delta}\left(D^{m} f(z)\right)}-\beta\right]>0, \quad \text { for }|z|<r_{\beta}
$$

where $r_{\beta}$ is given by (3.10).

Hence $\mathcal{L}_{a}^{\delta}\left(D^{m} f(z) \in S^{*}\left(\frac{1+(1-2 \beta) z}{1-z}\right)\right.$, for $|z|<r_{\beta}$, where $r_{\beta}$ is given by (3.10).

\section{Conclusion}

In the present work, we have introduced subclasses of analytic functions, which are generalization of many well-known classes. Several inclusion and inverse inclusion properties in terms of certain linear operators are discussed. We have proved preserving properties for these subclasses under convex convolution. Also, various applications of these results are deduced by using generalized Janowski functions and conic domains.

\section{References}

[1] H.A. Al-Kharsani and A. Sofo, Subordination results on harmonic $k$-uniformly convex mappings and related classes, Comput. Math. Appl. 59 (2010), 3718-3726. https://doi.org/10.1016/j.camwa.2010.03.071

[2] V.V. Anh and P.D. Tuan, On $\beta$-convexity of certain starlike functions, Rev. Roumaine Math. Pures App. 25 (1979), 1413-1424. 
[3] S. D. Bernardi, Convex and starlike univalent functions, Trans. Amer. Mat. Soc. 135 (1969), 429-446. https://doi.org/10.1090/S0002-9947-1969-0232920-2

[4] S.H.Z. Bukhari, K.I. Noor and B. Malik, Some applications of generalized Srivastava-Attiya integral operator, Iran. J. Sci. Technol. Trans. A Sci. (First Online) (2017) 1-7.

[5] N.E. Cho, The Noor integral operator and strongly close-to-convex functions, $J$. Math. Anal. Appl. 238 (2003), 202-212. https://doi.org/10.1016/S0022-247X(03)00270-1

[6] N.E. Cho and J.A. Kim, Inclusion properties of certain subclasses of analytic functions defined by a multiplier transformation, Comput. Math. Appl. 52 (2006), 323-330.

https://doi.org/10.1016/j.camwa.2006.08.022

[7] J. Dziok and K.I. Noor, Classes of analytic functions related to a combination of two convex functions, J. Math. Inequal. 11 (2017), 413-427.

https://doi.org/10.7153/jmi-2017-11-35

[8] W. Janowski, Some extremal problems for certain families of analytic functions, Ann. Polon. Math. 28 (1973), 297-326.

https://doi.org/10.4064/ap-28-3-297-326

[9] S. Kanas and A. Wisniowska, Conic regions and k-uniform convexity, J. Comput. Appl. Math. 105 (1999), 327-336.

https://doi.org/10.1016/S0377-0427(99)00018-7

[10] S. Kanas and A. Wisniowska, Conic domain and starlike functions, Rev. Roumaine Math. Pures Appl. 45 (2000), 647-657.

[11] R.J. Libera, Some classes of regular univalent functions, Proc. Amer. Math. Soc. 16 (1965), 755-758. https://doi.org/10.1090/S0002-9939-1965-0178131-2

[12] S.S. Miller and P.T. Mocanu, Differential Subordinations: Theory and Applications, Marcel Dekker, Inc., New York, Basel, 2000.

https://doi.org/10.1201/9781482289817 
[13] K.I. Noor, Applications of certain operators to the classes related with generalized Janowski functions, Integral Transforms Spec. Funct. 21 (2010), 557-567.

https://doi.org/10.1080/10652460903424261

[14] K.I. Noor, On classes of analytic functions defined by convolution with incomplete beta functions, J. Math. Anal. Appl. 307 (2005), 339-349.

https://doi.org/10.1016/j.jmaa.2004.10.043

[15] K.I. Noor, On a generalization of uniformly convex and related functions, Comput. Math. Appl. 61 (2011), 117-125.

https://doi.org/10.1016/j.camwa.2011.03.084

[16] K.I. Noor, On new classes of integral operators, J. Natur. Geom. 16 (1999), 71-80.

[17] K.I. Noor, M. Arif and W. Ul-Haq, On $k$-uniformly close-to-convex functions of complex order, Appl. Math. Comput. 215 (2009), 629-635.

https://doi.org/10.1016/j.amc.2009.05.050

[18] K.I. Noor and M.A. Noor, On certain classes of analytic functions defined by Noor integral operator, J. Math. Anal. Appl. 281 (2003), 244-252.

https://doi.org/10.1016/S0022-247X(03)00094-5

[19] K.I. Noor and M.A. Noor, On integral operators, J. Math. Anal. Appl. 238 (1999), 341-352.

https://doi.org/10.1006/jmaa.1999.6501

[20] K.I. Noor and S. Riaz, Application of Carlson Shaffer operator in univalent function theory, Acta Univ. Apulensis Math. Inform. 44 (2015), 175-188.

[21] H. Orhan, E. Deniz and D. Raducanu, The Feketo-Szego problem for subclasses of analytic functionsdefined by a differential operator related to conic domains, Comput. Math. Appl. 59 (2010), 283-295.

https://doi.org/10.1016/j.camwa.2009.07.049

[22] B. Pinchuk, Functions with bounded boundary rotation, Israel J. Math. 10 (1971), 6-16.

https://doi.org/10.1007/BF02771515

[23] Y. Polatoglu, M. Bolcal, A. Sen and E. Yavuz, A study on the generalization of Janowski functions in the unit disc, Acta Math. Acad. Paedagog. Nyhazi. (N.S.) 22 (2006), 27-31. 
[24] S. Ruscheweyh, New criteria for univalent functions. Proc. Amer. Math. Soc. 49 (1975), 109-115. https://doi.org/10.1090/S0002-9939-1975-0367176-1

[25] S. Ruscheweyh and T. Sheil-Small, Hadamard product of Schlicht functions and the Polya-Schoenberg conjecture, Comment. Math. Helv. 48 (1973), 119-135. https://doi.org/10.1007/BF02566116

[26] S. Ruscheweyh and V. Singh, On certain extremal problems for functions with positive real part, Proc. Amer. Math. Soc. 61 (1976), 329-334. https://doi.org/10.1090/S0002-9939-1976-0417847-4

[27] N. Xu and D. Yang, A class of analytic functions defined by Carlson-Shaffer operator, Bull. Inst. Math. Acad. Sin. (N.S.) 2 (2007), 91-102.

Khalida Inayat Noor

COMSATS University, Islamabad, Pakistan

e-mail: khalida@gmail.com

Shujaat Ali Shah

COMSATS University, Islamabad, Pakistan

and

Quaid-e-Awam University of Engineering, Science and Technology, Nawabshah, Pakistan

e-mail: shahglike@yahoo.com

This is an open access article distributed under the terms of the Creative Commons Attribution License (http://creativecommons.org/licenses/by/4.0/), which permits unrestricted, use, distribution and reproduction in any medium, or format for any purpose, even commercially provided the work is properly cited. 